\title{
TOWARD A COMPACT INSTRUMENT FOR DETECTING DRUG PRECURSORS IN DIFFERENT ENVIRONMENTS
}

\author{
F.Terzi ${ }^{1}, \quad$ A.Ulrici ${ }^{2}$, R.Seeber ${ }^{1}, \quad$ A.Secchi ${ }^{3}, \quad$ A.M.Fiorello ${ }^{3}$, M.Dispenza $^{3}$, \\ J.C.Antolín ${ }^{4}$, T.Kuusela ${ }^{5}$, A.Varriale ${ }^{6}$, S.D'Auria ${ }^{6}$, I.Tittonen ${ }^{7}$, F.Colao,

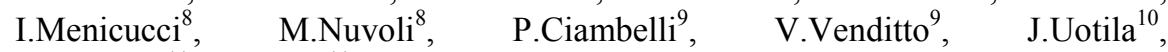 \\ G.Maisons $^{11}$,M.Carras ${ }^{11}$ \\ ${ }^{1}$ Dipartimento di Chimica and ${ }^{2}$ Dipartimento di Scienze Agrarie e degli \\ Alimenti, Università di Modena e Reggio Emilia, Italy; ${ }^{3}$ Selex-SI, Italy; \\ ${ }^{4}$ TECNALIA Research \& Innovation, Spain; ${ }^{5}$ Department of Physics and \\ Astronomy, University of Turku, Finland; ${ }^{6}$ IBP-CNR, Napoli, Italy; ${ }^{7}$ Department \\ of Micro- and Nanosciences Aalto University, Helsinki, Finland; ${ }^{8}$ ENEA, \\ Department UTAPRAD-DIM, Frascati (RM),Italy; ${ }^{9}$ Dipartimento di Ingegneria \\ industriale and Centre NANO_MATES, Università di Salerno, Fisciano $(\mathrm{Sa})$, \\ Italy; ${ }^{10}$ Gasera Ltd., Turku, Finland; ${ }^{11}$ III-V lab, Palaiseau, France
}

\begin{abstract}
Law enforcement agencies world-wide are keenly aware that chemical control is a crucial element to monitor the manufacture and distribution of illegal narcotics and synthetic substances. For this purpose one need components able to perform chemical identifications in contexts such as custom offices are needed, where inspection of trucks, cars, containers, as well as people and baggage is required.

Such components should be assembled as simple machine to be employed on-field by custom officers with no specific skill.

In the following the last results of the project CUSTOM will be presented. The work has been supported by the European Commission under the 7th Framework Programme through the Project SEC-242387: "Drugs and Precursors Sensing by Complementing Low Cost Multiple Techniques" (CUSTOM).
\end{abstract}

\section{Introduction}

A large number of different non-invasive hand held chemical sensors available in the market have been developed in the last decades. All of them have not been developed specifically for the drug precursors detection, but rather for explosive, drugs and chemical threats detection. They are mainly 
based on single approach, whether Ion Mobility Spectrometry (IMS), Surface Acoustic Wave (SAW), Gas Chromatography or Raman Spectroscopy.

The project CUSTOM aims to build up a compact and easy to use system able to provide screening over a large number of compounds, such as ephedrine, safrole, acetic anhydride and BMK, and discriminate them with low false alarm (FA) and high probability of detection (POD).

It is based on two complimentary approaches in order to identify an optimum trade-off between opposite requirements (compactness, simplicity, low cost, high sensitivity, low false alarm rate, selectivity). Those approaches are: a sensitive, selective, compact and low weight Laser Photo-Acoustic Spectroscopy (LPAS) for the first alarm, and a low cost, high data throughput fluorescent proteins based biochip for the fine screening.

The sensing platform is equipped with an air sampling system, including a special pre-concentrator module.

The components and subsystems are integrated in a standalone detector, which integrates the electronic board for the power generation and management, the data elaboration and signal processing, the communication interfaces and the micro-fluidic circuits for the gas samples addressing.

\section{Laser Photoacoustic spectrometer}

The initial prototype of the L-PAS sensor (see Figure 1) is a $320 \mathrm{~mm} \times 141$ mm x 184 mm module, which consists on a Quantum Cascade Laser (QCL), a photoacoustic cell and the readout interferometer[1].

The laser for the final prototype is a widely tunable External Cavity Quantum Cascade Laser (EC-QCL) delivering up to $200 \mathrm{~mW}$ of output power in the MID-IR range $\left(1200 \mathrm{~cm}^{-1}\right)$ with $200 \mathrm{~cm}^{-1}$ of tunability.

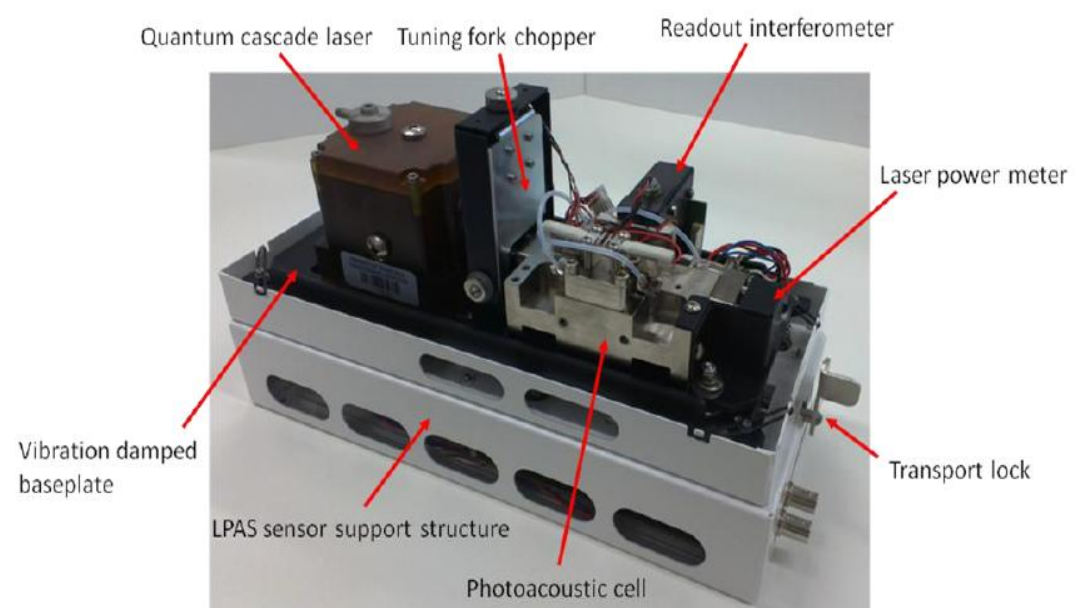

Figure 1-Layout of the LPAS module. 
A complex strategy of spectral response simulation has been developed in order to select the optimal wavelengths range. For this purpose different gas spectra have been collected from literature databases, denoised by means of the Wavelet Transform and mixed together according to a concentration matrix.

A specially developed single crystal Silicon On Insulator (SOI) based cantilever, is used as pressure sensor. The displacement of the cantilever is measured by a spatial type optical interferometer.

\section{Fluorescent proteins based biochip}

The first prototype of the fluorescent module is based on a competitive immunoassay [2] mechanism. It combines an immunochemical approach to bind the target analytes with a variation of fluorescence intensity by means of the Förster Resonance Energy Transfer (FRET) method.

Förster resonance energy transfer (FRET) is a process that relies on the energy transfer between two fluorophores: a donor and an acceptor.

The target compound contained in the sample competes to bind a specific antibody (donor) with a derivative (acceptor) of the target compound. Due to their importance in the drug precursors illegal trafficking, the first considered target analytes are the 1-Phenyl-2-propanone (BMK) and the Ephedrine.

One of the main tasks for the realization of the fluorescence sensor is the production of the reagents for the detection of the target compounds, namely anti ephedrine and anti BMK antibodies and ephedrine and BMK and their derivatives.

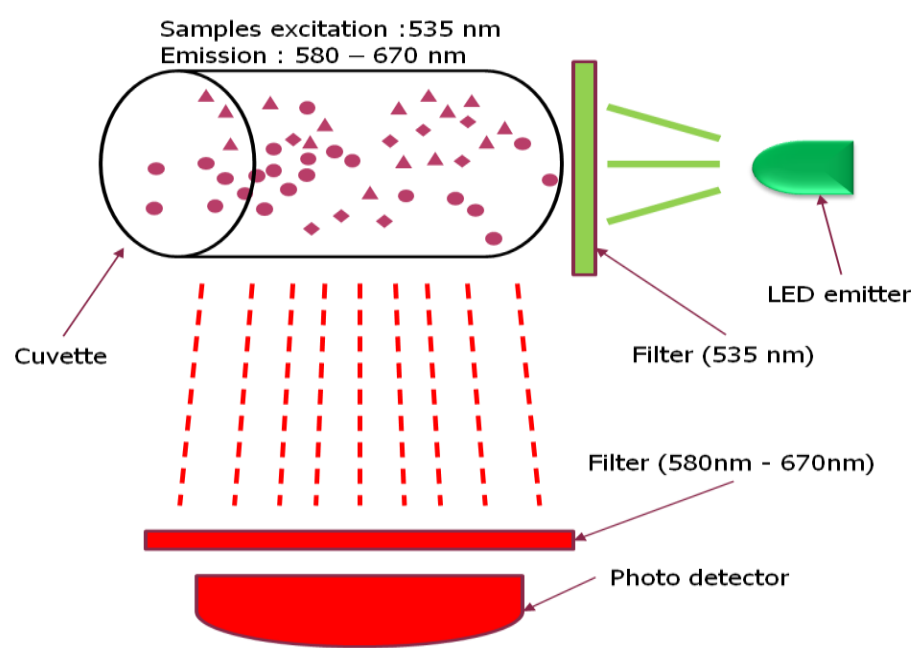

Figure 2 - Principle of operation of the fluorescence sensor 


\section{Preconcentrator}

The Pre-Concentrator (PC) is based on a nanoporous polymer material: the syndiotactic polystyrene (sPS), due its high absorption and desorption kinetics with the Volatile Organic Compound (VOC). It is in form of aerogels beads with features of 500-1000 microns. The preconcentrator consists on a serpentine path of $500 \mathrm{~mm}$ length and of $2 \mathrm{~cm}^{3}$ of volume filled with sPs. If thermal cycles are applied upon the PC, it shows absorption of the VOC at low temperature $\left(-10^{\circ} \mathrm{C}\right)$, and desorption at higher concentrations at higher temperature $\left(50^{\circ} \mathrm{C}\right)$.

The hardware used for the FRET reading out, whose scheme is depicted in Figure 2, consists mainly on a modulated LED light source in the green region to excite the fluorophores, a Photo Multiplier Tube (PMT) detector in the 580$670 \mathrm{~nm}$ range to detect the emitted light and a set of passive optical filters. Preliminary experiment showed a factor of 12 of the concentration of the target analyte during desorption. The use of the sPS based pre-concentrator improves the sensitivity of the whole sensors as well as the detection limit and the selectivity.

\section{Conclusion}

In this paper, the work the development of the novel sensor for Drugs And drugs PreCUrsor was showed. The features and the preliminary characterizations of the key components of the sensor: the QCL-PAS, the fluorescence module and the chemical pre-concentrator unit have been presented as self standing devices.

In the remaining part of the project, after final integration of the sensor, more detailed tests will be conducted in laboratory and real environment.

\section{References}

1. J. Uotila, J. Raittila, I. Kauppinen and J. Kauppinen, "Sensitive analysis of trace gases by using cantilever enhanced photoacoustic cell combined with a quantum cascade laser" 6th International Conference on Advanced Vibrational Spectroscopy (ICAVS6), June 2011.

2. A.Varriale, S. D'Auria, "An immuno-based surface plasmon resonance biosensor for ephedrine detection", Sec.+ Def. Conf.Prague 19 -22 Sept (2011), SPIE paper 8189A-21

3. Vincenzo Venditto, "Nanoporous crystalline polymers with sulfonated amorphous phases: highly efficient vocs sorbent materials", XII Congresso Nazionale di Chimica dell'Ambiente e dei Beni Culturali 